Mon. Not. R. Astron. Soc. 000,1-?? () Printed 5 February $2020 \quad$ (MN LATEX style file v2.2)

\title{
Cloud fragmentation cascades and feedback: on reconciling an unfettered inertial range with a low star formation rate
}

\author{
Eric G. Blackman ${ }^{1 \star}$ \\ ${ }^{1}$ Department of Physics and Astronomy, University of Rochester, Rochester NY, 14627, USA
}

\begin{abstract}
Molecular cloud complexes exhibit both (i) an unfettered Larson-type spectrum over much of their dynamic range, whilst (ii) still producing a much lower star-formation rate than were this cascade to remain unfettered all the way down to star-forming scales. Here we explain the compatibility of these attributes with minimalist considerations of a mass-conserving fragmentation cascade, combined with estimates of stellar feedback. Of importance is that the amount of feedback needed to abate fragmentation and truncate the complex decreases with decreasing scale. The scale at which the feedback momentum matches the free-fall momentum marks a transition scale below most of the cascade is truncated and the molecular cloud complex dissipated. For a $10^{6} M_{\odot}$ GMC complex starting with radius of $\sim 50 \mathrm{pc}$, the combined feedback from young stellar objects, supernovae, radiation, and stellar winds for a GMC cloud complex can truncate the cascade within an outer free-fall time but only after the cascade reaches parsec scales.
\end{abstract}

Key words: ISM: clouds; galaxies: star formation; ISM: evolution; ISM: structure; ISM: jets and outflows

\section{INTRODUCTION}

Modeling molecular cloud structure and observed starformation rate (SFR) of the Galaxy is a complex task, involving a nonlinear interplay between self-gravity, turbulence and stellar feedback in molecular cloud evolution (e.g Matzner \& McKee 2000; Krumholz et al. 2006; McKee \& Ostriker 2007; Dobbs, et al. 2014; Krumholz et al. 2014; Krumholz, et al. 2018; Kim et al. 2019). The effort to identify simplifying principles can be constructive.

The hierarchical structure of molecular clouds is consistent with an origin from fewer large clouds subjected to turbulent perturbations that facilitate fragmentation to smaller scales (Hoyle 1953; Chieze 1987; Field et al. 2008; Hopkins 2013). Predictions for mass, number, and density of molecular clouds as a function of scale emerge from this framework. As applied to the Galaxy, the outer scale corresponds to the largest giant molecular clouds (GMC) of radii $\gtrsim 50 \mathrm{pc}$ that source fragmentation into a quasi-self similar complex of clouds down to $\sim 0.1$ pc clump scales. Hereafter, "cloud complex (CC)" refers to the entire structure of subunits that emerges from an outer scale cloud.

The connection between a fragmentation cascade and the need for stellar feedback is as follows: the total mass

* E-mail: blackman@pas.rochester.edu of molecular gas in the ISM is approximately $2 \times 10^{9} M_{\odot}$. Most of this is contained in $\sim 2000$ of the most massive GMC (Combes 1991), implying a mass $M_{L} \sim 10^{6} M_{\odot}$ per complex. Taking the characteristic radius of these largest clouds to be $R_{L} \sim 50 \mathrm{pc}$, the average mass density is then $\rho_{L} \sim 1.6 \times 10^{22} \mathrm{~g} / \mathrm{cm}^{3}$. Without kinetic support, the clouds would collapse on a free fall time $t_{f f, L}$, which for a homogeneous sphere, is

$t_{f f, L}=\left(\frac{3 \pi}{32 G \rho_{L}}\right)^{\frac{1}{2}} \sim 5.5 \times 10^{6}\left(\frac{\rho_{L}}{1.6 \times 10^{-22} \mathrm{~g} / \mathrm{cm}^{3}}\right)^{-\frac{1}{2}} \mathrm{yr} .(1)$

If star formation were $100 \%$ efficient and occurred on a time scale $t_{f f, L}$, then dividing cloud mass by this free fall time gives the unfettered "no-feedback" SFR (Zuckerman \& Evans 1974; McKee \& Ostriker 2007; Federrath 2015). For 2000 of these large GMC, the total Galactic SFR is then

$$
\begin{aligned}
\dot{M}_{n f, T}= & N \dot{M}_{n f} \sim 360\left(\frac{N}{2000}\right)\left(\frac{M_{L}}{10^{6} M_{\odot}}\right) \\
& \times\left(\frac{\rho_{0}}{1.6 \times 10^{-22} \mathrm{~g} / \mathrm{cm}^{3}}\right)^{-\frac{1}{2}} M_{\odot} / \mathrm{yr}
\end{aligned}
$$

where $\dot{M}_{n f}$ is the no-feedback SFR per large GMC. The observationally inferred Galactic SFR is $\dot{M}_{o b s} \sim 4 M_{\odot} / \mathrm{yr} \sim$ $\dot{M}_{n f, T} / 100$ van den Bergh 1991; Stahler \& Palla 2004; Diehl et al. 2006). 
Normalizing the SFR by mass and free fall time at a given scale $R$, we can construct a dimensionless ratio

$\epsilon_{f f}(R)=\frac{\dot{M}_{o b s} f_{R} t_{f f}(R)}{M_{R}}$

where $f_{R}$ is the fraction of star formation occurring within structures of scale $R$, where $R$ ranges from $R_{L}$ down to clump scales, $M_{R}$ is the sum of the mass in all structures with scale $R$ (later made explicit in equation (6) ), and $t_{f f}$ is the free fall time for structures of scale $R$. Equation (3) is inspired by equation (1) of Krumholz \& Tan (2007):

$S F T_{X-f f}=\frac{\dot{M}_{o b s} f_{X} t_{f f, X}(R)}{M_{X}}$

where $f_{X}$ is the fraction of galactic star formation occuring in objects of some specified class $X, M_{X}$ is the total mass class $X$ objects in the Galaxy, and $t_{f f, X}$ is their free fall time. Were we to allow "objects of class $X$ " to represent "objects of scale $R$," then equations (3) and (4) could be the same. Krumholz \& Tan (2007) do not use $X$ in equation (4) to primarily distinguish objects by scale, but by observation type. Krumholz et al. (2014) compiled and reviewed data indicating that $S F T_{X-f f} \simeq 0.01$, independent of density of the object class. Inasmuch as objects of a given scale in the cascade also correspond to objects of a given density, there is some correspondence between equations (3) and (4).

Here $\epsilon_{f f}$ can be immediately seen to be constant for a mass conserving fragmentation cascade: First, $\dot{M}_{o b s}$ is an observed constant. Second, the ratio of $\frac{t_{f f}(R)}{M_{R}}$ is just the inverse of an unfettered constant mass throughput through the cascade that reflects the mass conserving assumption. Third, since the cloud complex forms a nested structure with smaller clouds embedded in larger clouds, all star formation that occurs within the nested cloud structure is contained in all larger clouds, making $f_{R}$ in equation (3) is also constant.

We will discuss how, in the fragmentation cascade, a low SFR $\epsilon_{f f}<<1$ is consistent with unfettered fragmentation over most of the observed "inertial range" down to a transition scale. This follows because the feedback needed to abate the cascade decreases with decreasing scale and because combined feedbacks primarily abate the cascade at small truncation scales. There is cognizance of the potential influence of different feedbacks on different scales (e.g Kim et al. 2019), but here we pithily combine a fragmentation cascade with feedbacks to estimate a transition scale.

In section 2, we discuss the conceptual picture and quantitative scalings of the fragmentation cascade, including the predicted number spectrum and size-velocity-density relations. In section 3 , we show quantitatively that the momentum (and energy) of feedback needed to abate the fragmentation cascade and reduce the SFR is a function of scale. In section 4, we discuss and combine feedbacks from young stellar objects (YSO) and supernovae (SN) and radiation and winds from massive stars. We find they can truncate the cascade by the end of a typical outer scale free fall time, but only after the cascade survives unfettered to pc scales. In section 5, we comment on how the feedback is consistent with supplying $H I$ back to the ISM and supplying a substrate of turbulent fluctuations for the next incarnation of fragmentation. We conclude in section 6 .

\section{MASS CONSERVING CASCADE}

\subsection{Virial equipartition and basic framework}

Molecular clouds with radial scales 50pc down to clump scales $\sim 0.5 \mathrm{pc}$ were long thought to obey the Larson scaling relations for velocity dispersion $\sigma \propto R^{1 / 2}$ and density $\rho(R) \propto R^{-1}$ respectively (Larson 1981; Solomon et al. 1987), so that $M(R) \propto R^{2}$. The constants of proportionality were seemingly consistent with virial equipartition $\alpha_{v} \sim 1$ where $\alpha_{v} \equiv \frac{3 G M}{5 \sigma^{2} D}$ is a minimalist virial parameter (Bertoldi \& McKee 1992), for spherical clouds. However, Hever et al. (2009) found that updated measurements are more consistent with $\sigma \propto \Sigma^{1 / 2} R^{1 / 2}$ and $\alpha_{v}<1$, with the surface density $\Sigma$ varying in different regions. Others suggest that that the velocity dispersion may be primarily infall. Vázquez-Semadeni, et al. 2008; Ballesteros-Paredes et al. 2011, 2012). Nearly 90\% of the Hever et al. (2009) cloud sizes are larger than $1 \mathrm{pc}$.

Field et al. (2011) suggested that the Heyer et al. (2009) results may still be consistent with clouds in VE but only if (i) external pressure is taken into account and (ii) clouds are at a "critical surface density" (Chieze 1987; Elmegreen 1989) which minimizes the equlibrium value of $\sigma^{2} / R$. This is obtained by extremizing the generalized $\mathrm{VE}$ equation

$\frac{\sigma^{2}}{R}=\frac{1}{3}\left(\pi \Gamma G \Sigma+\frac{4 P_{e}}{\Sigma}\right)$

with respect to $\Sigma$ to find $\Sigma_{c}=13.9\left(\frac{P_{e} / \Gamma}{10^{5} \mathrm{erg} / \mathrm{cm}^{3}}\right)^{1 / 2} \mathrm{~g} / \mathrm{cm}^{2}$, where $P_{e}$ is the external pressure, $\Gamma$ accounts for cloud density structure $(\Gamma=3 / 5$ for uniform density; $\Gamma=0.73$ for isothermal sphere). Plugging $\Sigma_{c}$ into Eq. (5) and solving for $R$ then gives $R=R_{c} \propto \sigma^{2}$. Complementarily, replacing $\Sigma$ by $\Sigma_{c}$ in Eq. (5) and writing $R=\left(\pi \Sigma_{c} / M\right)^{-1 / 2}$ and solving for $M$ gives $M=M_{c} \propto \sigma^{4} \propto R^{2}$. These proprotionalities also follow if we use $\Sigma \equiv M / 4 \pi R^{2}$ and instead extremize equation (5) with respect to $R$. For constant $P_{e}$ in a given complex, Larson-type scalings therefore emerge if clouds are at their critical values. Explaining the full range of clouds requires $P_{e}$ to vary by 2 orders of magnitude between regions, but gravity from a shell of HI (Elmegreen 1989) is a gravitational alternative to external pressure.

Clouds might naturally appear at their critical values because the critical mass varies as $\sigma^{4}$, and so once the largest scale cloud forms, its fragmentation can only occur after supersonic $\sigma$ decays enough, likely via shocks, (Keto et al. 2019) for the fixed cloud mass to be of order twice the critical mass. As subunits collapse and establish quasi-virial equilibrium, these subunits would themselves be close to the marginally unstable critical masses for the next stage in the cascade, and so on. If fragmentation into two subunits were somehow to occur before a cloud mass were twice the critical mass, then at least one of the fragments would be rapidly unstable and shorter lasting that a critical cloud of that same mass. Therefore the probability of seeing clouds near critical would be higher than seeing sub or super-critical clouds.

All that said, the precision to which VE of clouds can be determined from observations remains low (Singh, Matzner \& Jumper 2019). Clouds also deviate from the spherical symmetry commonly assumed. Nevertheless, 
Fragmentation Cascade and Feedback

we push forward and assume that the plausibility arguments above can justify use of observed Larson-type scalings.

Initial fragmentation likely requires that clouds not only have sufficient gravity but also pre-existing density fluctuations because free fall times vary as $\rho^{-1 / 2}$. Density fluctuations as high as $30 \%$ over background may be needed to facilitate subunits to collapse before the overall cloud does (Toalá et al. 2015). Plausibly, these fluctuations can arise from interacting supersonic velocity fluctuations whose energy is supplied by a combination of gravity itself, and externally, e.g.. SN driven, turbulence Mac Low \& Klessen 2004; Kritsuk, Lee \& Norman 2013a; Padoan et al. 2016; Kritsuk, Ustyugov \& Norman 2017).

We self-consistently ignore magnetic fields for present purposes on the following grounds: magnetic fields in galaxies originate from kinetic energy from velocity flows. On the $\sim 50 \mathrm{pc}$ scales from which the largest GMC form and initiate the fragmentation cascade, $\rho v_{T}^{2} \geq \rho v_{A}^{2}$. If Larson-type relations hold, then $\rho v_{T}^{2} \sim$ constant, so this inequality is maintained throughout the fragmentation inertial range. Debate remains (e.g. Federrath 2015; Krumholz \& Federrath 2019), but observations at least do not reveal dynamically dominant magnetic fields above core scales Crutcher 2012; Nixon and Pringle 2019). The concepts herein apply whether magnetic fields are at or below turbulent equipartition.

\subsection{Mass spectrum}

The total mass contained in clouds of radius $R$ within a nested complex is (Field et al. 2008)

$M_{R}=\int_{0}^{x} M\left(x^{\prime}\right) \frac{d N}{d x^{\prime}} d x^{\prime}=\int_{0}^{M(R)} M^{\prime} \frac{d N}{d M^{\prime}} d M^{\prime}$

where $x \equiv R / R_{L}, R_{L}$ and $M_{L}=\int_{0}^{1} M\left(x^{\prime}\right) \frac{d N}{d x^{\prime}} d x^{\prime}$ are the outer (largest) scale GMC radius and mass, $d N / d x^{\prime}$ and $d N / d M^{\prime}$ are the number of clouds within the scale range $d x^{\prime}$ and mass range $d M^{\prime}$ respectively.

Mass conservation though an unfettered cascade in which critical clouds evolve on a dynamical, or approximate free fall time, means that that $\dot{M}_{R} \simeq M_{R} / t_{f f}(R)=$ constant for all $R$. Using cloud scaling relations from Sec. 2.1 we have $M \propto R^{2}$ and thus

$t_{f f}(R) \propto \rho^{-1 / 2} \propto(R / M(R))^{3 / 2} \propto R^{1 / 2} \propto M^{1 / 4}$.

Dividing equation (6) by $t_{f f} \propto M^{1 / 4}$ and taking $d M \sim M$ gives $M^{7 / 4} d N / d M=$ constant, or

$d N / d M \propto M^{-7 / 4}$.

This is consistent with the observed range $1.3 \leq d \log N / d \log M \leq 1.9$ (Mvers 1983; Casoli. Combes \& Gerin 1984; Solomon et al. 1987; Loren 1989; Williams, Blitz \& Stark 1995; Snell, Carpenter \& Heyer 2002; Blitz, et al. 2007). Since $M \propto x^{2}$, equation (8) is also equivalent to

$\frac{d N}{d x} \propto x^{-2.5}$.

That observations down to clump scales are roughly consistent with these spectra and the Larson-type relations highlights a consistency with the unfettered mass fragmentation cascade. But observations also require $\epsilon_{f f}<<1$. This can be explained if star-formation is blocked by cascadeabating feedback only on relatively small scales. This explanation depends on the fact that less feedback momentum is required to abate the cascade at small scales than large scales, as discussed in the next section. Some mass continues its cascade below the truncation scale to supply star formation, and can still exhibit a similar $d N / d x$ spectra on core scales (Lada, Bally \& Stark 1991), but with a coefficient that would reflects the volume filling fraction (and thus mass fraction) that avoided feedback.

\section{SCALE DEPENDENT MOMENTUM AND ENERGY CASCADE RATES}

For a mass conserving fragmentation cascade in each GMC complex, the no-feedback scalar momentum deposition rate on scale $R$ from collapse is

$$
\begin{aligned}
& \frac{d P_{g}}{d t}(R)=\rho v_{f f}^{2} \cdot 4 \pi R_{L}^{2} x^{2} \cdot x d N / d x \simeq 4 \pi \rho_{L} G M_{L} R_{L} x^{1 / 2} \\
& \simeq 3.5 \times 10^{31} x^{1 / 2}\left(\frac{M_{L}}{10^{6} M_{\odot}}\right)^{2}\left(\frac{R_{L}}{50 \mathrm{pc}}\right)^{-2} \mathrm{~g} . \mathrm{cm} / \mathrm{s}^{2},
\end{aligned}
$$

where we have used equations (7), (9) $M_{L}=10^{6} M_{\odot}, R_{L}=$ $50 \mathrm{pc}$, and the scale independence of $\rho v_{f f}^{2}$ implied by Larson's relations to replace this latter quantity by $\rho_{L} v_{f f, L}^{2}$, where $v_{f f}$ and $v_{f f, L}$ are the free fall speeds for arbitrary scale $R$ and scale $R_{L}$ respectively. Similarly, the energy deposition rate is

$$
\begin{aligned}
& \frac{d E_{g}}{d t}(R)=\rho v_{f f}^{3} \cdot 4 \pi R_{L}^{2} x^{2} \cdot x d N / d x \\
& \simeq 4 \pi \rho_{L} G^{3 / 2} M_{L}^{3 / 2} R_{L}^{1 / 2} x \\
& \simeq 3.3 \times 10^{37} x\left(\frac{M_{L}}{10^{6} M_{\odot}}\right)^{5 / 2}\left(\frac{R_{L}}{50 \mathrm{pc}}\right)^{-1.5} \mathrm{~g} \cdot \mathrm{cm}^{2} / \mathrm{s}^{3},
\end{aligned}
$$

where have also used $v_{f f} \propto R^{-1 / 2}$. Equations (10) and (11) show that the free fall energy and momentum feedback requirements to abate the cascade and reduce SFR are less demanding on smaller scales. Abating the free fall momentum (energy) flux is the more (less) stringent requirement if whatever supplies the feedback has a larger (smaller) speed than free fall on the feedback coupling scale. Below we focus on momentum feedback.

\section{TRANSITION SCALE AND FEEDBACK}

From equation (10), the required momentum for abating a fragmentation cascade at $R \sim 0.5 \mathrm{pc}$ for example, is 10 times smaller than at $R=R_{L}=50 \mathrm{pc}$. Feedback that can only truncate the fragmentation cascade after it proceeds to these small scales would not threaten unfettered Larsontype relations at larger scales. Feedback from low mass stars is dominated by YSOs, and feedback from massive stars comes from SN, winds, and radiation (Krumholz et al. 2014; Kim \& Ostriker 2015; Iffrig \& Hennebelle 2015; Kim et al. 2019; Grudić, et al. 2019). We consider each in turn below. Given these feedback sources, we then determine the maximum transition scale below which the fragmentation is abated.

\subsection{YSO Feedback}

Matzner \& McKee (2000) found theoretically that YSOs can deliver $\sim 40 \mathrm{~km} / \mathrm{s}$ of momentum per unit mass of stars 
formed. Simulations of Carroll et al. (2009) are consistent with similar values. For one of our 2000 outer scale GMC, the SFR is $2 \times 10^{-3} \sim M_{\odot} /$ yr over its $5.5 \times 10^{6}$ yr free fall time, so there is then

$P_{Y}=5.5 \times 10^{6} \cdot 2 \times 10^{-3} M_{\odot} \cdot 40 \mathrm{~km} / \mathrm{s}=8.8 \times 10^{43} \mathrm{~g} . \mathrm{cm} / \mathrm{s}(12)$

of momentum delivered by YSOs. The latter is also consistent with observational studies Quillen, et al. 2005; Brunt, Heyer \& Mac Low 2009; Nakamura, et al. 2011) suggesting that stellar outflows can inject a turbulent velocity dispersion for typical molecular clouds $\sim 1.5 \mathrm{~km} / \mathrm{s}$ at $10 \%$ volume filling fraction at $\sim 0.2 \mathrm{pc}$ scales. 1

\subsection{SN Feedback}

For a $10^{6} M_{\odot}$ GMC, and a stellar mass spectrum of $d N / d m \propto m^{-2.3}$ with stellar mass range $0.1 \leq m \equiv$ $M_{*} / M_{\odot} \leq 100$ Chabrier 2003), there would be

$N\left(m_{0}\right)=N_{0} \int_{m_{0}}^{100} m^{-2.3} d m$,

stars of $m>m_{0}$ producing $\mathrm{SN}$ during a time scale $t_{f f, L}$, where $N_{0}=1721$ is the normalization that ensures $N_{0} M_{\odot} \int_{m_{0}}^{100} m^{-1.3} d m=10^{4}$ or $10^{6}\left(\epsilon_{f f} / 0.01\right) \sim 10^{4}$ for a free fall time $t_{f f, L}$. Since only stars with $m_{0} \geq 40$ could contribute over this time scale (Maeder \& Meynet 1987), we obtain $N\left(m_{0}=40\right) \sim 7$. These SN would inject an initial momentum $P_{S N, I}\left(M_{*}\right) \simeq 7 \sqrt{\left(2 \frac{M_{*}}{2 M_{\odot}} \frac{E}{10^{51} \mathrm{erg} / \mathrm{s}}\right)}=$ $6.3 \times 10^{43} \mathrm{~cm} / \mathrm{s}$, for $M_{*}=40 M_{\odot}$ (assuming ejected mass $\sim M_{*} / 2$ for $\left.M_{*}>M_{\odot}\right)$ and $E=10^{51} \mathrm{erg}$. But during the Sedov phase, approximately $\sim 8$ times more momentum is injected from the work done by the post shock gas (Iffrig \& Hennebelle 2015; Kim \& Ostriker 2015; Martizzi, et al. 2016). We therefore obtain the momentum contribution

$P_{S N} \simeq 8 P_{S N, I}\left(40 M_{\odot}\right)=5 \times 10^{44} \mathrm{~g} \cdot \mathrm{cm} / \mathrm{s}$.

There is also a contribution from background SN: most Galactic SN occur within the inner $5 \mathrm{kpc}$ thin disc, of thickness of $\sim 300 \mathrm{pc}$ or volume $2.4 \times 10^{10} \mathrm{pc}^{3}$. Since our 2000, $50 \mathrm{pc}$ outer scale GMC's have volume $\simeq 10^{9} \mathrm{pc}^{3}$, they fill just $4 \%$ of the total region. If we put $m_{0} \sim 8$ in equation (13), we obtain the total of $32>>2$ stars that produce SN from a given cloud complex, even if that complex has disappeared. Assuming that the complexes do not last more than a a time scale $t_{f f, L}$ (justified later), then most $\mathrm{SN}$ will not occur in existing complexes. However, averaged over time scales $>>10 \mathrm{Myr}$, some of these SN will be randomly cospatial with existing GMC. For SN rate $1 / 40 \mathrm{yr}$ (Tammann, Loeffler \& Schroeder 1994; Li et al. 2011) there would be roughly $(1 / 40 \mathrm{yr})(0.04 / 2000)\left(5.5 \times 10^{6} \mathrm{yr}\right) \sim 3 \mathrm{such}$ background SN per complex free-fall time contributing to the feedback. Their momentum contribution is

$P_{S N, b} \sim 3 P_{S N}\left(10 M_{\odot}\right)=1.4 \times 10^{44} \mathrm{~g} \cdot \mathrm{cm} / \mathrm{s}$.

1 Observationally, outflow feedback may be underestimated by principal component analysis (PCA): Carroll et al. (2010) showed that PCA, when used to identify turbulent driving scales (Brunt, Heyer \& Mac Low 2009), is biased toward low amplitude large-scale velocity structures, even if energetically subdominant.

\subsection{Radiation Feedback}

Theory and simulation conclude that per unit mass of star formation, radiation supplies $\sim 200 \mathrm{~km} / \mathrm{s}$ of momentum (e.g Kim et al. 2019), beginning shortly after the first stars of a complex form and mostly due to ionizing radiation. The consequent momentum deposited in a complex free fall time is then

$P_{R}=5.5 \times 10^{6} \cdot 2 \times 10^{-3} M_{\odot} \cdot 200 \mathrm{~km} / \mathrm{s}=4.4 \times 10^{44} \mathrm{~g} . \mathrm{cm} / \mathrm{s} .(16)$

\subsection{Winds from Massive Stars}

The momentum imparted per star from massive stellar winds is $P_{w, *}=M_{e} v_{\infty} \leq M_{*} v_{\infty}$ where $M_{e}\left(M_{*}\right)$ is the mass ejected over the entire the active wind before $\mathrm{SN}$ and $v_{\infty} \lesssim 2.5 v_{e s}=3 \sqrt{\left(G M_{*} / R\right)^{1 / 2}}$ is the terminal wind speed Kudritzki \& Puls 2000). Using $R / R_{\odot} \sim 1.3\left(M_{*} / M_{\odot}\right)^{3 / 5}$, (Demircan \& Kahraman 1991) then $v_{e s}(m) \simeq 5.36 \times$ $10^{7} \mathrm{~m}^{1 / 5} \mathrm{~cm} / \mathrm{s}$. For the total momentum contribution integrated over the mass function of massive stars, we divide the contribution into (i) the most massive stars that eject $\sim 1 / 2$ or more their mass and $v_{\infty}=2.5 v_{e s c}$, and (ii) slightly lower mass stars which eject $\sim 0.1$ of their mass with $v_{\infty} \sim 1.5 v_{\text {esc }}$ during the wind loss phase. We then have

$$
\begin{aligned}
& P_{W} \simeq \epsilon_{W} N_{0} M_{\odot}\left[1.25 \int_{20}^{100} m^{-1.3} v_{e s} d m+0.15 \int_{10}^{20} m^{-1.3} v_{e s} d m\right] \\
& =4.1\left(\epsilon_{W} / 0.15\right) \times 10^{43} \mathrm{~cm} . \mathrm{g} / \mathrm{s},
\end{aligned}
$$

where $\epsilon_{W}$ is an efficiency factor. That equation (17) with $\epsilon_{W}=1$ is comparable to the contrinution from SN is consistent with previous detailed work (e.g. Fierlinger, et al. 2016), but is likely an over-estimate because hot gas from winds may escape an inhomogeneous environment: Rosen et al. (2014) estimate $\epsilon_{W} \sim 0.15$.

\subsection{Total Feedback and the Transition Scale}

Combining equations (12) (14) (15), (16), and (17) gives the total momentum delivered over an outer GMC free-fall $t_{f f, L}$ as

$P_{T}=P_{Y}+P_{S N}\left(M_{* 0}\right)+P_{S N, b}+P_{R}+P_{W}=1.2 \times 10^{45} \mathrm{erg} / \mathrm{s} .(18)$

Setting this equal to equation (10) multiplied by $t_{f f, L}=$ $5.5 \mathrm{Myr}$ gives the scale of equality of $x=0.044$, or $R=2.2 \mathrm{pc}$. Thus, the combination of these momentum feedbacks during a time $t_{f f, L}$ could truncate the cascade and dissipate the complex below these scales. This is qualitatively consistent with the destruction seen in simulation movies (e.g Grudić, et al. 2019)). The trickle of star formation arises gas that does make it through to smaller scales. The mass spectrum for this subset of mass that avoids the feedback ad continues to fragment may remain similar to that above the transition scale, but normalized by a much smaller filling fraction.

During the time scale of $t_{f f, L}$ the $7 \mathrm{SN}$ from the complex cascade and the 3 background SN would inject $\sim 10^{52} \mathrm{erg}$ into the complex. Ionizing radiation would inject photons at rate of $\sim 10^{50} / \mathrm{s}$ (McKee \& Williams 1997) for an energy input $>3.6 \times 10^{53} \mathrm{erg}$. The amount of energy needed to unbind all the $H_{2}$ in the gas of the complex is about $9 \times 10^{52}$ erg, so these sources do have enough energy to unbind the 
Fragmentation Cascade and Feedback

molecules at $3 \%$ efficiency. Thus, once the cascade is abated, much of the gas may be returned to the ISM as HI.

\section{RESEEDING TURBULENCE AND HI}

SN are a source of galactic turbulence for much of the ISM (Spitzer 1978; Norman \& Ferrara 1996), supplemented by a substrate of shear driven turbulence (Sellwood \& Balbus 1999). This also seeds turbulence in molecular clouds (Mac Low \& Klessen 2004; Padoan et al. 2016). The latter is important, as discussed in section 2. since a source of subunit density fluctuations within a parent cloud is needed to seed subunit fragmentation. In addition, feedback from SN and radiation can convert gas into HII which cools into HI. This can also account for the observed mass of HI in shells, energy content of shells and supershells, and depletion of $\mathrm{H}_{2}$ between spiral arms. We discuss each below.

Heiles (1979) found that most of the atomic hydrogen is contained within 45 shells (defined by energy content $<$ $\left.3 \times 10^{52} \mathrm{erg}\right)$ and 18 supershells with larger energies. The latter dominate both the shell masses and energies by factors of 10 to 100 . The total HI mass in shells is about $M_{H I} \sim$ $4 \times 10^{8} M_{\odot} \cdot 2$ Heiles (1979) estimated a typical age of $t_{s b} \sim$ $10^{7} \mathrm{yr}$ for the lifetime of HI supershells, so the needed rate of HI supply would be $\sim M_{H I} / t_{s b}=40 M_{\odot} /$ yr. Dividing the typical SN injected momentum of $\sim 5.1 \times 10^{43} \mathrm{~g} \cdot \mathrm{cm} / \mathrm{s}$ (for a $10 M_{\odot}$ progenitor including the factor of 8 discussed in the previous section) by typical flow speeds $\sigma_{S N} \sim 10 \mathrm{~km} / \mathrm{s}$ when remnants break up gives a mass influenced per SN of $M \sim$ $2.5 \times 10^{4} M_{\odot}$. Multiplying by the galactic SN rate $1 / 40 \mathrm{yr}$, then gives $625 M_{\odot} / \mathrm{yr}$, so only $6.4 \%$ of this need be involved to account for the observations even if we just appeal to SN. Dviding the total kinetic energy $\sim 2 \times 10^{54} \mathrm{erg}$ in observed shells (Heiles 1979) by typical lifetimes $t_{s b} \sim 10^{7} \mathrm{yr}$, gives a required energy injection rate $6.7 \times 10^{39} \mathrm{erg} / \mathrm{s}$, which can also be accomplished by SN during that time scale, which inject $10^{51} \times 0.02 / \mathrm{yr} / 3 \times 10^{7} \mathrm{sec} / \mathrm{yr}=6.6 \times 10^{41} \mathrm{ergs}$.

Finally note that the bulk of the total $\sim 2 \times 10^{9} M_{\odot}$ of $\mathrm{H}_{2}$ in the Galaxy resides in spiral arms. The absence of $\mathrm{H}_{2}$ in the inter-arm regions means that the molecular gas must be converted into $\mathrm{HI}$ by the time that material moves through the arms. At radii of $5 \mathrm{kpc}$, there are $\sim 4$ arms (Vallee 2005) each of which take $\sim 1.3 \times 10^{8}$ yr to pass through as the gas motion has a significant component along the arms. This lengthens the time scale for gas to pass through the arms from the value of $\sim 1 / 4$ of a rotation period for purely radial arms. The minimum rate of $\mathrm{HI}$ formation needed to account for the absence of $\mathrm{H}_{2}$ in the inter-arm regions is then $\sim \frac{2 \times 10^{9} M_{\odot}}{1.3 \times 10^{8} \mathrm{yr}} \sim 15.4 M_{\odot} /$ yr. But since feedback destroys the complex on an outer overturn time, the supply rate can be as high as $\sim \frac{2 \times 10^{9} M_{\odot}}{5.5 \times 10^{6} \mathrm{yr}} \sim 360 M_{\odot} /$ yr so this is fast enough.

\section{CONCLUSIONS}

We have discussed how molecular cloud complexes, interpreted as mass-conserving fragmentation cascades, have

2 The total HI mass in the Galaxy is closer to $4 \times 10^{9} M_{\odot}$ Dickev \& Lockman 1990; Sparke \& Gallagher 2006) properties roughly consistent with an unfettered cascade with clouds near their critical unstable mass over most of their inertial range, while still producing star formation with low efficiency. This can work because the feedback momentum needed to abate the cascade and destroy the cloud complex decreases with decreasing scale. The primary sources of feedback momentum available from YSOs, and massive stars provide enough momentum and energy to abate the cascade on a free-fall time of the outer complex scale, and dissipate the complex, but only after the fragmentation cascade reaches pc scales. In this way, the star formation is damped while the super-transition scale inertial range retains Larson-type relations and an unfettered size spectrum power law consistent with observations. Below the transition scale, there will be some mass that slips through the feedback and this goes onto form stars. For that gas, the number-size spectrum may be retained, but with a normalization coefficient that is reduced to reflect participation of only the mass fraction that beats the feedback.

Pinning down the existence and value of the transition scale, or using the framework as a guide to help interpret observations may be useful. The complexity of the interaction between feedback, fragmentation, and turbulence has and continues to warrant more detailed calculations and simulations, but the goal of synthesizing various pieces into an overall framework remains a conceptually important complement to improved understanding of the microphysics.

\section{ACKNOWLEDGMENTS}

Thanks to G. Field for related discussions on clouds, supershells, and HI, and to E.Keto for particuarly useful comments on the manuscript. I acknowledge support from NSF Grant AST-1813298, KITP (UC Santa Barbara) funded by NSF Grant PHY-1748958, and the Aspen Center for Physics funded by NSF Grant PHY-1607611.

\section{REFERENCES}

Blitz L., Fukui Y., Kawamura A., Leroy A., Mizuno N., Rosolowsky E., 2007, prpl.conf, 81, prpl.conf

Brunt C. M., Heyer M. H., Mac Low M.-M., 2009, A\&A, 504,883

Cabrit S., \& Bertout C., 1992, A \& A., 261,274

Casoli F., Combes F., Gerin M., 1984, A\&A, 133, 99

Ballesteros-Paredes, J., D'Alessio, P., Hartmann, L. 2012. MNRAS 427, 2562.

Ballesteros-Paredes, J., Vázquez-Semadeni, E., Gazol, A., Hartmann, L. W., Heitsch, F., Colín, P. 2011. MNRAS 416, 1436.

Bertoldi, F., \& McKee, C. F. 1992, ApJ, 395, 140

Carroll J. J., Frank A., Blackman E. G., Cunningham A. J., Quillen A. C., 2009, ApJ, 695, 1376

Carroll J. J., Frank A., Blackman E. G., 2010, ApJ, 722, 145

Chabrier G., 2003, PASP, 115, 763

Chieze J. P., 1987, A\&A, 171, 225

Combes, F. 1991, ARAA, 29, 195

Crutcher R. M., 2012, ARA\&A, 50, 29

Demircan O., Kahraman G., 1991, Ap\&SS, 181, 313 
Dickey J.M. \& Lockman F.J., 1990, ARAA, 28215.

Diehl, R., et al. 2006, Nature, 439, 45

Dobbs, C. L.; Krumholz, M. R.; Ballesteros-Paredes, J.; Bolatto, A. D.; Fukui, Y.; Heyer, M.; Low, M. -M. M.; Ostriker, E. C.; Vzquez-Semadeni, E., 2014, in Protostars and Planets VI, Henrik Beuther, Ralf S. Klessen, Cornelis P. Dulle mond, and Thomas Henning (eds.), (University of Arizona Press, Tucson), p.3,

Elmegreen B. G., 1989, ApJ, 338, 178

Federrath C., 2015, MNRAS, 450, 4035

Field, G. B., Blackman, E. G., Keto, E. R. 2008, MNRAS 385, 181-188.

Field, G. B., Blackman, E. G., Keto, E. R. 2011, MNRAS 416, 710-714.

Fierlinger K. M., et al., 2016, MNRAS, 456, 710

Grudić, M. Y., Hopkins P. F., Lee E. J., Murray N., Faucher-Giguère C.-A., Johnson L. C., 2019, MNRAS, 488, 1501

Guszejnov D., Hopkins P. F., 2016, MNRAS, 459, 9

Guszejnov D., Hopkins P. F., Krumholz M. R., 2017, MN-

RAS, 468, 4093

Guszejnov D., Hopkins P. F., Grudić M. Y., 2018, MNRAS, 477,5139

Harper-Clark E., Murray N., 2009, ApJ, 693, 1696

Heiles C., 1979, ApJ, 229, 533

Heyer M., Krawczyk C., Duval J., Jackson J. M., 2009, ApJ, 699, 1092

Hopkins P. F., 2013, MNRAS, 430, 1653

Hoyle F., 1953, ApJ, 118, 513

Iffrig O., Hennebelle P., 2015, A\&A, 576, A95

Keto E. R., Field, G.B., Blackman E.G. 2019, in press, MNRAS.

Kim C.-G., Ostriker E. C., 2015, ApJ, 802, 99

Kim J.-G., Kim W.-T., Ostriker E. C., 2019, ApJ, 883, 102

Kritsuk A. G., Lee C. T., Norman M. L., 2013, MNRAS, 436, 3247

Kritsuk A. G., Ustyugov S. D., Norman M. L., 2017, NJPh, 19, 065003

Krumholz, M. R., Matzner, C. D., \& McKee, C. F. 2006, ApJ, 653, 361

Krumholz M. R., Federrath C., 2019, FrASS, 6, 7

Krumholz, M. R., \& Tan, J. C. 2007, ApJ, 654, 304

Physics Reports 539, 49-134.

Krumholz, M. R et al. 2014., in Protostars and Planets VI 243.

Krumholz M. R., Burkhart B., Forbes J. C., Crocker R. M., 2018, MNRAS, 477, 2716

Kudritzki R.-P., Puls J., 2000, ARA\&A, 38, 613

Lada E. A., Bally J., Stark A. A., 1991, ApJ, 368, 432

Larson, R. B. 1981, MNRAS, 194, 809

Li, W., Chornock, R., Leaman, J., Filippenko, A. V., Poznanski, D., Wang, X., Ganeshalingam, M., Mannucci, F. 2011. Nearby supernova rates from the Lick Observatory Supernova Search - III. MNRAS,412, 1473.

Loren R. B., 1989, ApJ, 338, 902

Mac Low, M.-M., \& Klessen, R. S. 2004, Reviews of Modern

Physics, 76, 125

Maeder A., Meynet G., 1987, A\&A, 182, 243

Martizzi D., Fielding D., Faucher-Giguère C.-A., Quataert E., 2016, MNRAS, 459, 2311

Matzner C. D., McKee C. F., 2000, ApJ, 545, 364

McKee, C. F., \& Williams, J. P. 1997, ApJ, 476, 144
McKee C. F., Ostriker E. C., 2007, ARA\&A, 45, 565

Myers, P. C. 1983, ApJ, 270, 105

Nakamura F., et al., 2011, ApJ, 726, 46

Nixon, C. J., Pringle, J. E. 2019. New Astronomy 67, 89.

Rahner D., Pellegrini E. W., Glover S. C. O., Klessen R. S., 2017, MNRAS, 470, 4453

Rosen, A. L., Lopez, L. A., Krumholz, M. R., \& Ramirez-

Ruiz, E. 2014, MNRAS, 442, 2701

Quillen A. C., et al., 2005, ApJ, 632, 941

Norman C. A., Ferrara A., 1996, ApJ, 467, 280

Padoan P., Pan L., Haugbølle T., Nordlund Å., 2016, ApJ, 822,11

Sellwood J. A., Balbus S. A., 1999, ApJ, 511, 660

Singh A., Matzner C. D., Jumper P. H., 2019, ApJ, 878, 22

Snell R. L., Carpenter J. M., Heyer M. H., 2002, ApJ, 578, 229

Solomon, P. M., Rivolo, A. R., Barrett, J., \& Yahil, A. 1987, ApJ, 319, 730

Sparke L.S. \& Gallagher J.S., 2006, Galaxies in the Universe - 2nd Edition, (Cambridge University Press: Cambridge)

Spitzer, L. 1978. Physical processes in the interstellar medium, (New York: Wiley)

Stahler S. \& Palla F., 2004, in Formation of Stars, (Wiley, New York).

Shirley, Y. L., Evans, N. J., II, Young, K. E., Knez, C., \& Jaffe, D. T. 2003, ApJS, 149, 375

Tammann G. A., Loeffler W., Schroeder A., 1994, ApJS, 92,487

Tammann G. A., Loeffler W., Schroeder A., 1994, ApJS, 92, 487

Toalá J. A., Vázquez-Semadeni E., Colín P., Gómez G. C., 2015, MNRAS, 446, 3725

Vázquez-Semadeni E., González R. F., Ballesteros-Paredes J., Gazol A., Kim J., 2008, MNRAS, 390, 769

Vallée J.P., 2005, AJ, 130569.

van den Bergh S., 1991, PhR, 204, 385

Williams J. P., Blitz L., Stark A. A., 1995, ApJ, 451, 252

Zuckerman, B., \& Evans, N. J. 1974, ApJL, 192, L149 